gargle, and nose-douche. In cases in which there bave been muscular and articular pains in addition to the catarrhal affection hot baths and massage have proved to be of great use. The best time for this sort of treatment, according to my experience, is during the months of May and June-that is to say, before the attacks of hay-fever have begun to show themselves. July I should not recommend; in August and September, 'however, I have treated cases with success. There are cases of hay-fever which do not respond to the treatment. These I have always found to be cases in which there was no history of gout, while in those cases in which the combination of gout and summer catarrh existed I have scarcely ever failed to obtain a good result. The following is the history of a few of my cases.

CASE 1.-The patient, a female, aged 19 years, had suffered from hay-fever for many years. There was a history of gout. The affection generally began towards the end of May. Hay, roses and other flowers, as well as heat and dust, immediately brought on an attack which began with sneezing and running at the eyes and nose, and which after a short time was followed by coughing. During June and July the attacks were of almost daily occurrence. In other respects the patient was perfectly well. There was some thickening of the mucous membrane of the nose, especially on the left side. Bleeding from the nose was easily brought on. The treatment as described above was carried out in April and May, 1897, with the result that there were no attacks of hay-fever during that summer. A second shorter course of treatment was thought advisable in April, 1898. There has been no recurrence of the attacks of hay-fever up to the present time (March, 1900).

CASE 2.-The patient, a female, aged 36 years, had suffered from hay-ferer since the age of 15 years. Both her parents were gouty. The patient herself had suffered from rheumatic pains and articular swellings. She had severe attacks of hay-fever from May till August, recurring almost daily and much exhausting her. During the attacks there was great swelling of the upper part of the face, intense frontal headache, much secretion from the eyes and nose, together with coughing and a wheezing respiration. Local treatment of every description had been useless. Relief was only to be obtained by residing in a bracing place at the seaside. The patient was treated at Neuenahr during part of May and June, 1897, and though the attacks became less violent they did not entirely cease. The treatment was repeated in the following year with the result that no attacks of hay-fever occurred during that summer, nor has there been any recurrence since 1898, but I should mention that the patient has lately been more subject to rheumatic pains.

CASE 3.-In this case a history of gout was obtainable. The patient, a female, aged 42 years, was first treated for hay-fever at Nenenahr during 1895 with good results. She remained free from attacks during the following two years. In 1898 a relapse occurred which made her return to Neuenahr; she had after that no more attacks during 1898 and 1899.

CASE 4.-The patient, a girl, aged 10 years, came of a very gouty family and was herself a very delicate child. She has been suffering from severe attacks of hay-fever for the last four years. She was quite disfigured by the attacks. Her face was swollen and red, her eyes were inflamed, and there was constant running at the nose together with coughing and wheezing respiration. She contracted the attacks everywhere and from all sorts of causes, but especially from heat and dust. When she arrived at Neuenabr she was in the midst of one of her most severe attacks and was very prostrated. During the following four weeks she kept quite free from the attacks, nor did the long journey to St. Moritz affect her, although the weather was hot and dusty. She has since kept wonderfully well.

CasE 5.-This case is one of those treated without any success. The patient had suffered from hay-fever and asthma for several years. There was no history of gout. The attacks occurred almost daily during May and June. They began in the usual way with sneezing and increased nasal secretion, but their chief feature was violent coughing, a feeling of serere tightness round the chest, and a difficulty in breathing. The usual treatment was carried out but had to be given up after its ineffectiveness had been proved.

The following are the conclusions to which I am led. 1. There are cases of summer catarrh (so-called hay-fever) which can be cured, or at least relieved, by treatment with the waters of Neuenahr. 2. The cases especially suited for this treatment are those in which the affection is a part of the gouty dyscrasia. 3. Cases without a history of gout do not seem to be benefited by the treatment.

Neuenahr, Germany.

\section{EXERCISES IN THE TREATMENT OF LATERAL CURVATURE OF THE SPINE.}

By NOBLE SMITH, F.R.C.S. EDIN., L.R.C.P. LOND., SENIOR SURGEON TO THE CITY ORTHOP IDIC HOSPITAL, LONDON.

THE beneficial influence of gymnastic exercises upon the general health of the body has been recognised for ages and it is needless, in discussing the present subject, to dwell upon this fact further than to state that in treating curvatures of the spine everything which benefits the general health will materially assist other treatment.

My present purpose is to consider the local effects which may be produced upon a crooked spine by specified muscular exercises, either by correcting the shape of and strengthening the joints, or by strengthening any particular set of muscles connected with the deformity. In the first place it should be noted that it is impossible to lay down any hardand-fast rules for gymnastic exercises by which to deal with every instance of curvature, because cases differ to such an extent that what is suitable to one individual would be totally wrong for another. Moreover, patients differ as regards the muscles they bring into use in carrying out the same movements. I will in the first place describe briefly the various kinds of lateral curvature and the application of gymnastics to their peculiar requirements.

CLASS 1. - That in which the most notable condition is debility occurring after illness or from some other cause. In these cases the muscular system being especially affected the patient is unable to hold his spine erect except for short periods, but in the early stage there is not any well-marked bony deformity. For such a condition a course of general active and passive exercises of the trunk and neck, and also of the legs and arms, will have a beneficial effect. As a rule, these exercises should be carried out while the patient is in a recumbent position. The general health of these patients always requires treatment by means of a nutritious diet and tonic medicines, some form of lime being especially beneficial. Massage is also helpful. It is usually also advisable to limit the amount of voluntary active movements in order to avoid producing fatigue.

Class 2.- In this class we find, in addition to the above symptoms, laxity of the ligaments of the spinal joints. For these patients exercises will be beneficial, but greater care must be taken to prevent the spine from subsiding into curves during the intervals between the exercises.

CLASS 3.-In which alterations in the form of the bones has already occurred. In these cases the exercises must be directed not only generally, but specially, in order to overcome these curves. For instance, the special exercise to be presently described to correct rotation is one of the most important.

CLASS 4.-- In which the curves are more severe. For these patients some good may be done doubtless by a course of exercises, but less permanent effect must be hoped for than in the less severe cases.

In Class 1 mentioned above it is nearly always desirable to commence treatment by exercises and in many cases with the addition of massage. In Class 2, also, exercises may be carried out, but the patient should be carefully watched to determine whether he is really recovering and to detect when the disappearance of curves is more apparent than real. In Class 3 and Class 4 a combination of methods must be brought to bear upon the case.

Description of exercises. - A great variety of complicated exercising machines have been devised and used from an early date in the study of this deformity, but although these apparatus are very beneficial under some circumstances, it should be the object of the surgeon to simplify as far as possible the various movements carried out, with the view of teaching the patient to continue the treatment by himself as far as possible subsequently.

In the early periods of treatment of any case, however, the knowledge of the surgeon and the efforts of the skilled teacher are both necessary, and unless the curvature be very 
slight the medical supervision is reguired for a considerable time.

General exerciscs.-I have already stated that, as a rule, the patient should rest in the recumbent position whilst carrying out the general exercices. The object of the recum. bency is to remove the weight of the body from influencing the curres.

We almost invariably find that where there is general debility and a weak muscular system there also exists laxity of the joints. Under such circumstances whenever the spine is in an upright position, whether the patient be standing or sitting, there is a certain deforming influence upon these lax joints caused by the superincumbent weight, and the spine consequently tends to give way in one or other direction.

The object of general exercises is to strengthen the muscles and also, by bringing more blood to the weak parts, to nourish and strengthen the whole spine. The test as to when a patient may safely undertake exercises in an upright or sitting 1 os.tion must be his ability to control his spine in a straight posture for a sufficient length of time. For instance, when we have first examined the case the patient,

FIG. 1.

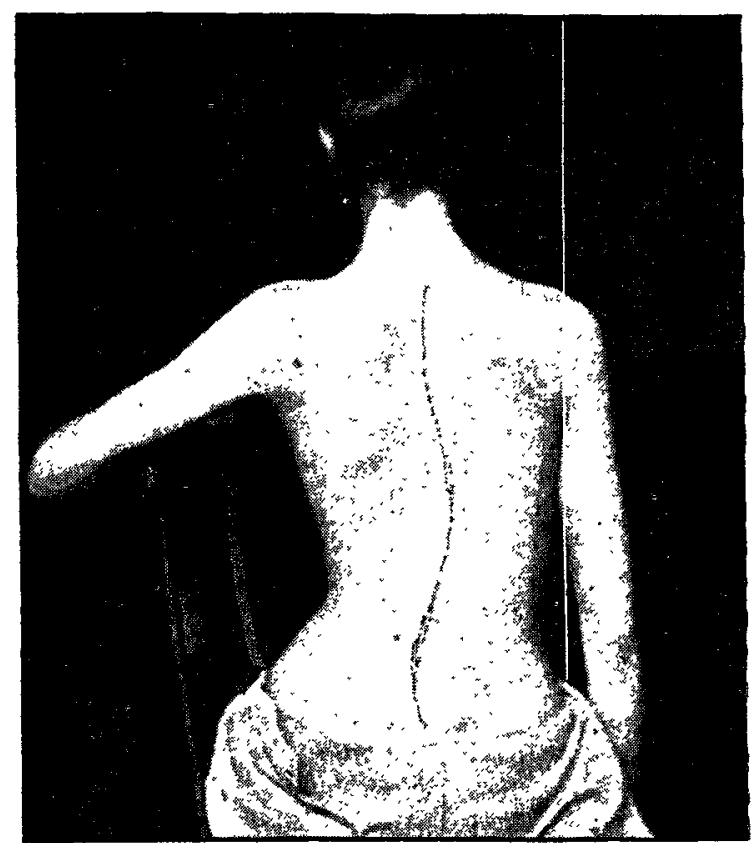

Dorso-lumbar curvature, the dorsal curve being most promir ent about the tenth dorsal vertebra.

FIG. 2.

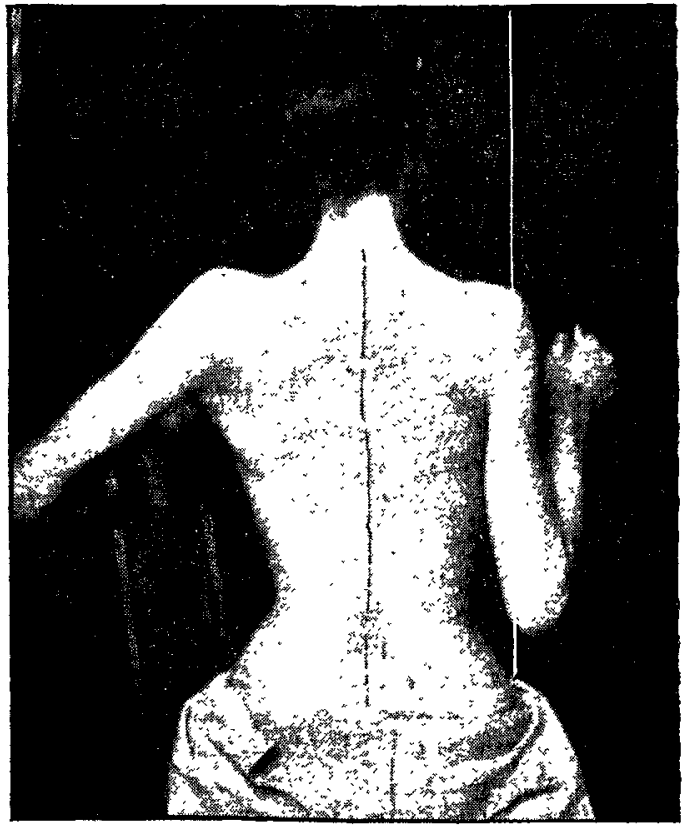

The same patient as in Fig. 1 drawing back the elastic expander with the right arm, tbus using the mutcles between the shoulder and the spinous processes of the deflected dorkal vertebræ, straightening the whole spine and un-rotating the vertebræ. The vertical lines from the outer side of the pelvis show the relatire positions of the side of the body, without and with the active exercise. upon sitting in a chair, will, after a certain short period, have relapsed as regards his spine into one or other or several curves. When the time has arrived that he can sit up easily with the spine in a perfectly normal position not only as regards lateral but also in respect to anteroposterior posture for at least five minutes, then we need not restrict the exercises to the recumbent position.

FIG. 3.

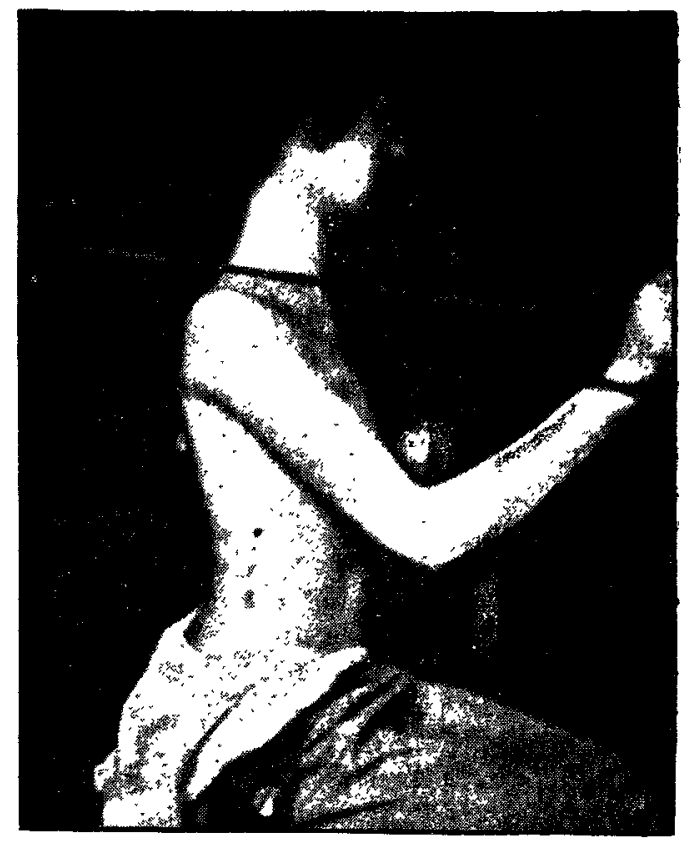

The exercise for drawing back the prominent side of the thorax. Here the dorsal curve was to the left. The patient in Fig. 1 and Fig. 2 would have done this exercise with the left arm.

FIG. 4.

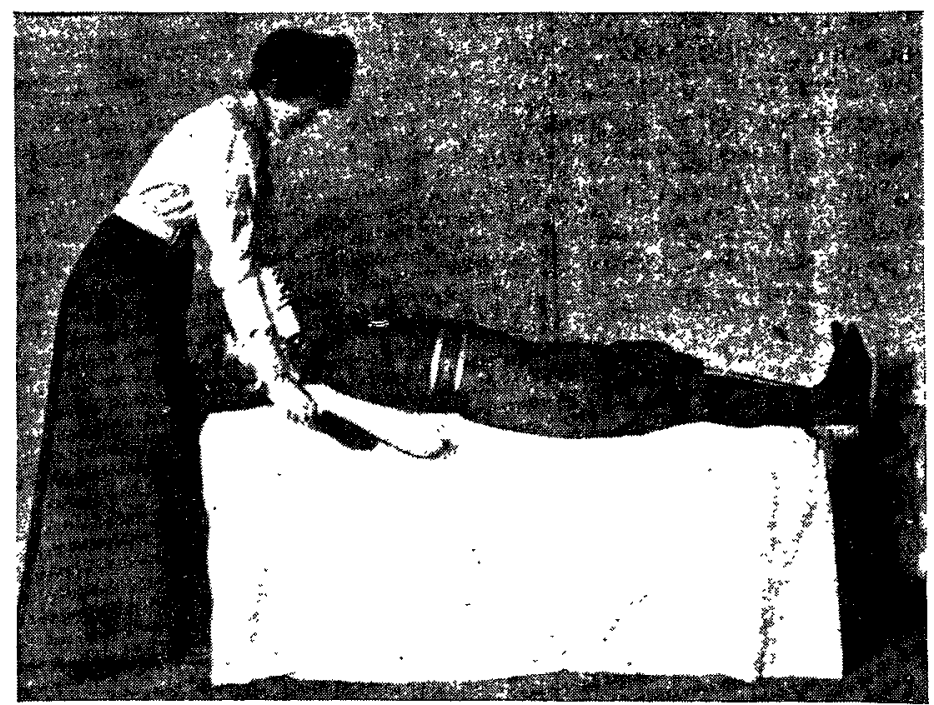

Arm extension, first position, with resistance by the patient. In the second position the arms $\varepsilon$ re raised and the operator resists.

FIG. 5.

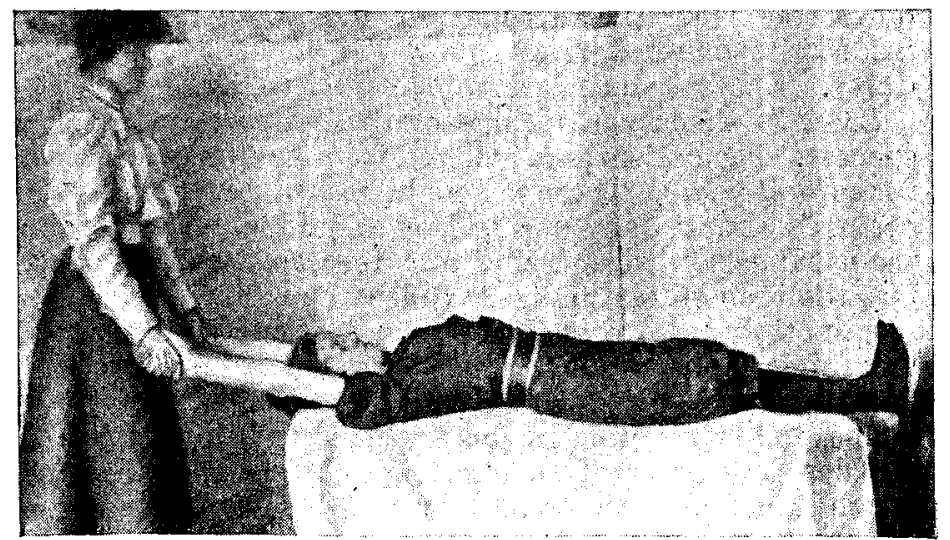

Arm extension, second position, in which the operator resists. 


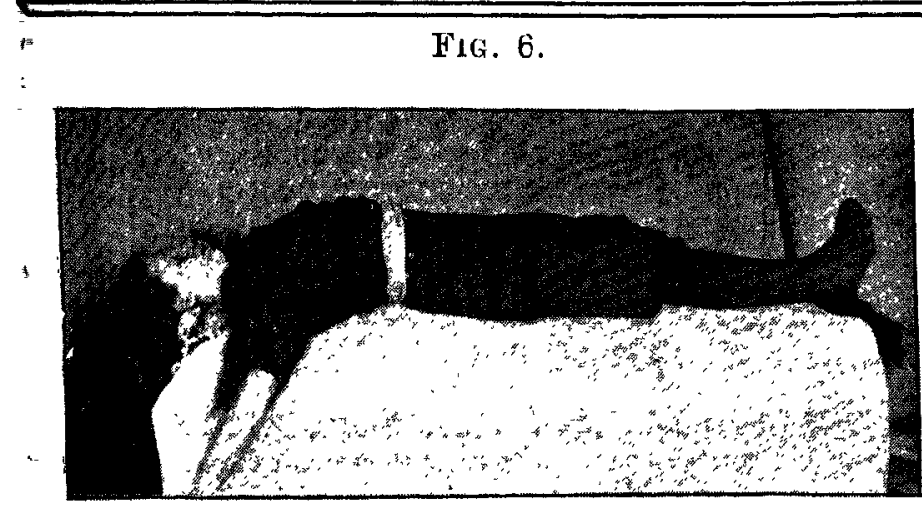

Arm extension without resistance, second rosition. In the first position the arms are flexed and the fingers touch the shoulders.

FIG. 7.

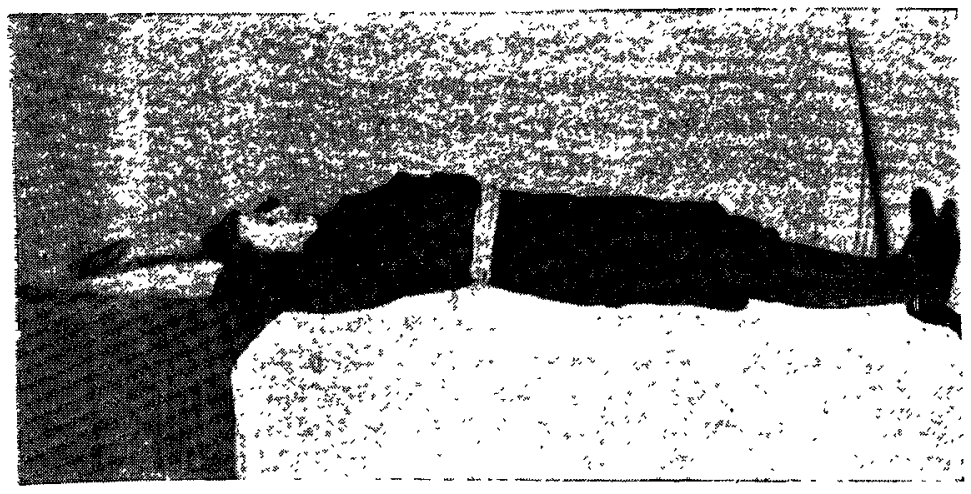

Arm extension, third position.

FIG. 8.

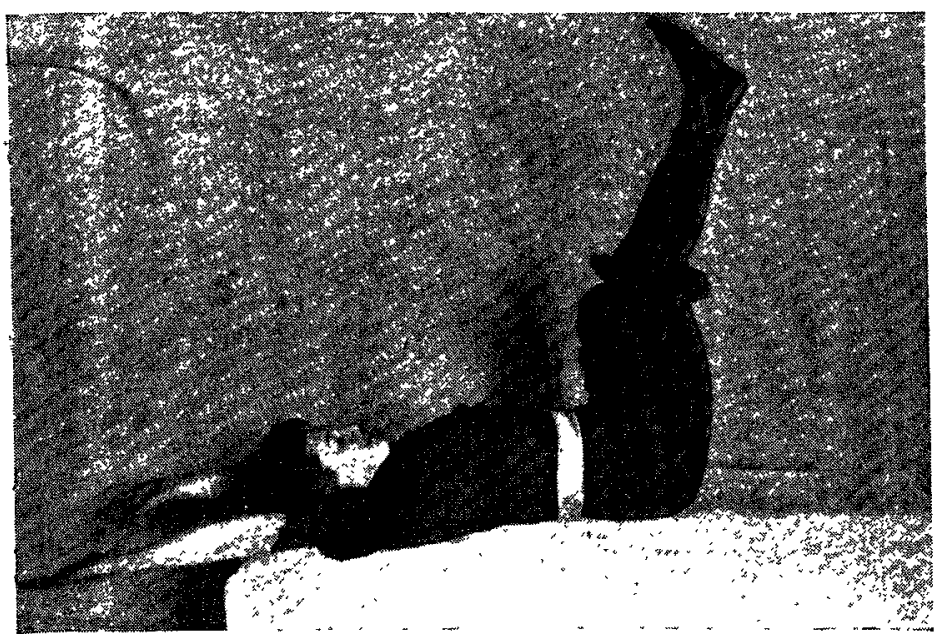

Simultaneous leg elevation. The arms are extended to balance the body. This exercise has a powerful effect upon the abdominal and lumbar muscles and has to be attained by gradual slighter movements.

FIG. 9.

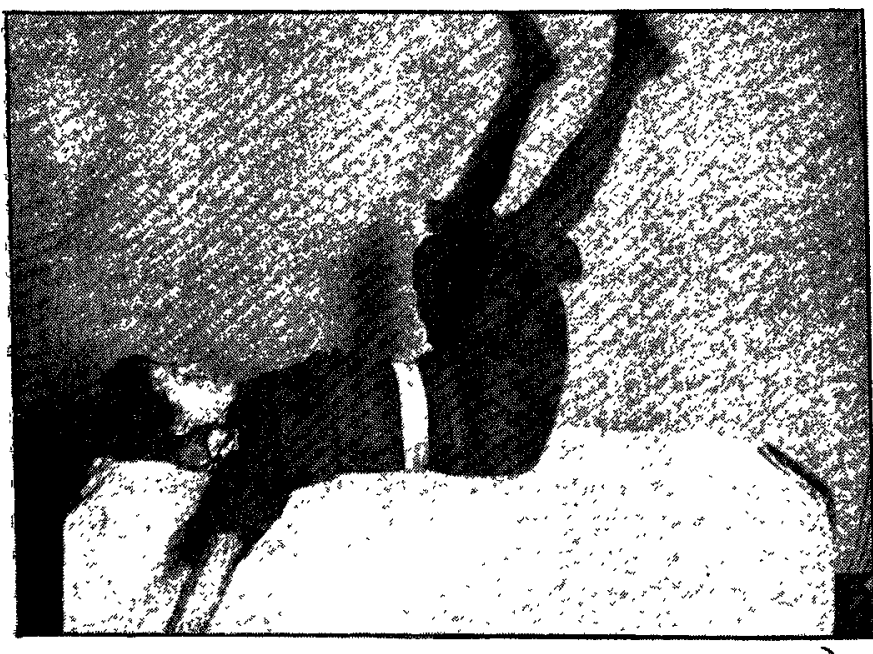

Simultaneous leg and arm circling. The combination of these exercises is only curried out in exceptional cases and requires considerahle strength.
FIG. 10 .

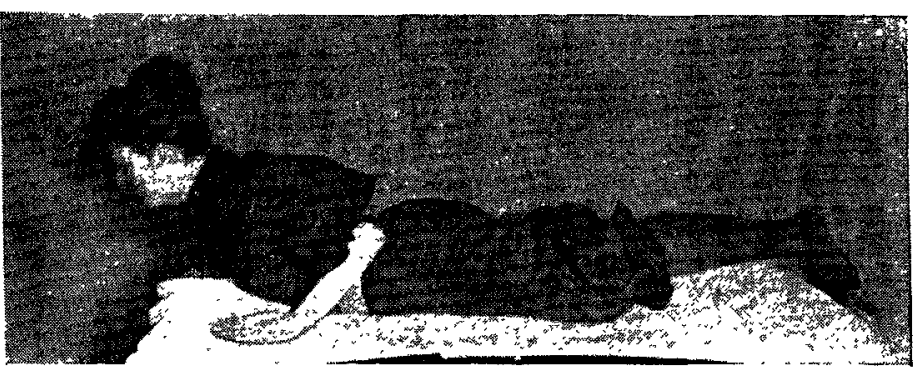

This figure shows the first position for the exercises in Fig. 11 and Fig. 12.

FIG. 11.

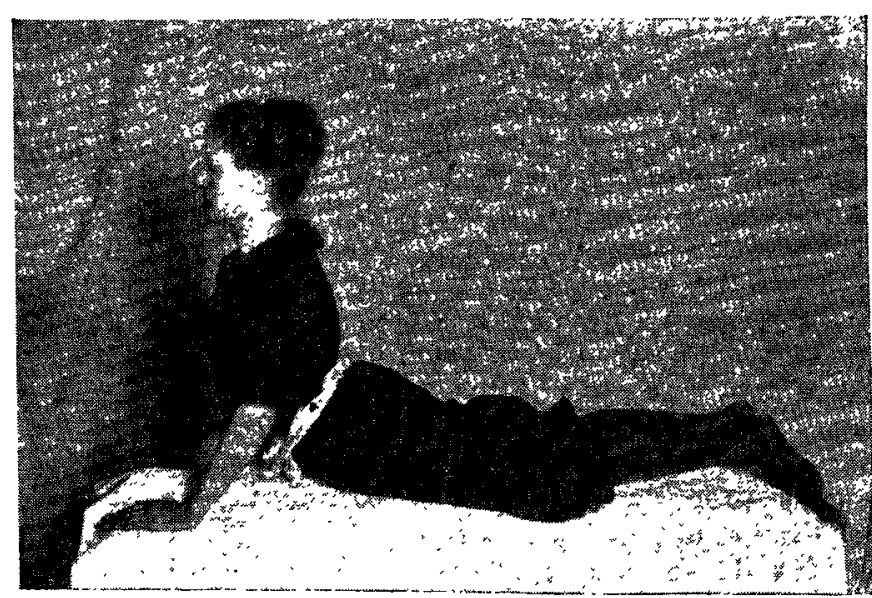

- Body flexion backwards and forwards. This exercise requires considerable practice before it can be done evenly to the extent shown.

FIG. 12.

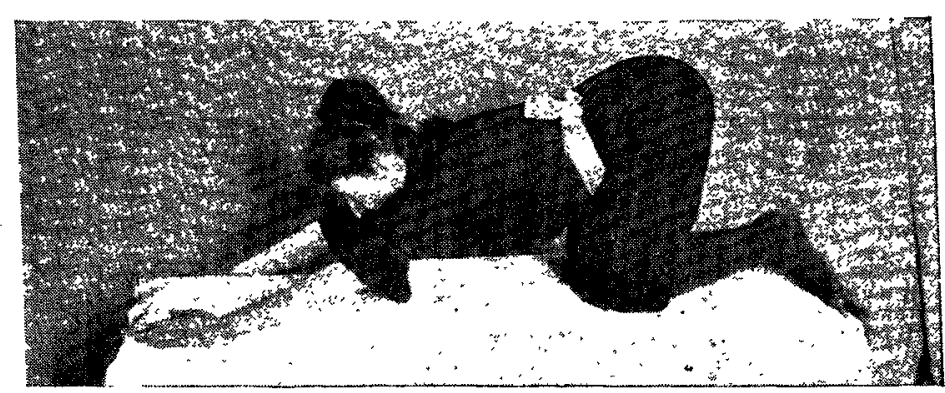

Kneeling body movement. It would require many figures to show the details of this exercise. Before it can be carried to be practised.

FIG. 12.

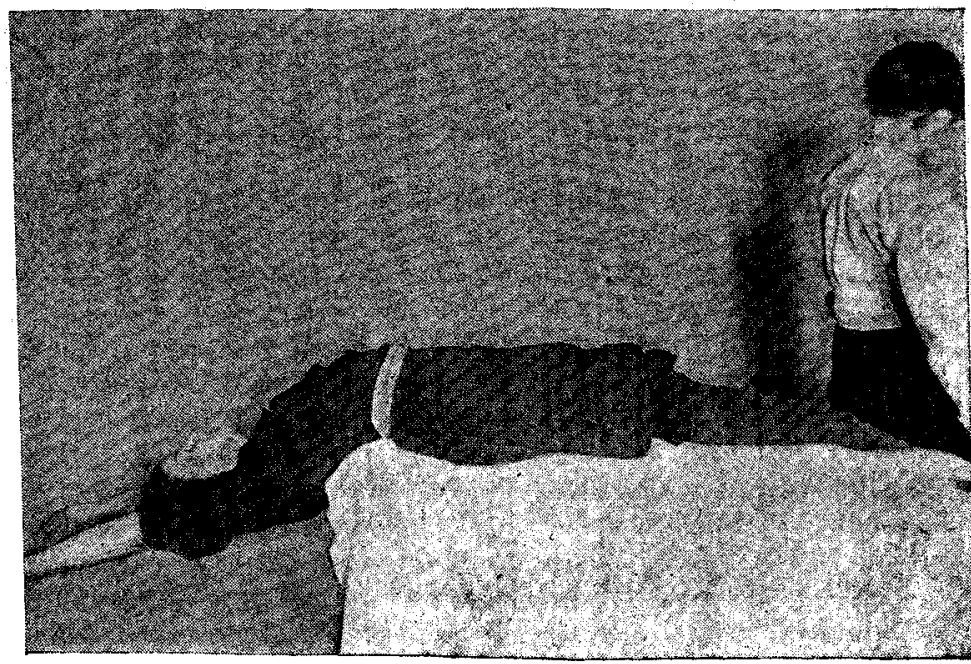

Backward bending with legs fixed. If forward bending to a sitting position is required the patient must have the knees bent over the edge of a shorter couch and there fixed. 
Exercises to act directly upon the curves.-In order thoroughly to realise the effect of exercises upon a curved spine it is necessary to consider carefully the exact action of the muscles which we propose to bring to our aid and the exact position of the parts which are distorted.

One exercise which has been recommended for acting directly upon the dorsal curve has been devised with the object of drawing ont the concave side of the curve by use of the arm of that side, but this exercise tends to increase the twisting of the spine which is the most serious part of the deformity.

The muscles which extend from the arm to the spine are the trapezius, the rhomboidei, and the latissimus dorsi and these are attached to the spinous processes of the vertebræ.

It should be remembered that the spinous processes are directed, in consequence of the rotation of the vertebræ, towards the side of the concavity and that the rotation is the part that offers the more serious obstruction to treatment. The effect of this exercise is obviously to draw the spinal processes further in the direction of the concavity and consequently to increase the rotation.

The plan of special exercises which I have devised for acting upon the dorsal rotation consists in the use (1) upon the convex side of the curve of the muscles above referred to with the object of drawing the vertebræ round towards their proper position; and (2) upon the concave side of the curve of the muscles which extend from the arm to the front of the body, with the object of drawing backwards that prominent side of the thorax. Although this plan of exercise acts directly only upon the dorsal curve, yet the resistance of the pelvis to the action of the arms tends to twist the lumbar part of the spine in the opposite direction and thus produces the exact action that is required. If more direct action is needed for a lumbar curve the quadratus lumborum may be brought into use upon the convex side of this curve, and this is best done with the patient in the recumbent position. The object of this system is to lessen the curves upon their convexities and to rotate the vertebræ back to their normal position.

Slight variations in these exercises must be made to suit individuai cases, as there is nearly always some difference in the form and condition of the curves. The general exercises of the erector spinæ muscles of both sides should, as a rule, be carried out in addition to the special exercises now described and avoidance of fatigue and suitable rest should be observed.

In the earlier stages of treatment the special exercises should be conducted while the patient is lying recumbent both in the supine and prone position, but it will perhaps suffice here to describe these exercises as carried out in the sitting position.

For this exercise upon the convexity a hook should, in the first instance, be placed, as a rule, on a level with the top of the patient's shoulder while sitting, but often it is necessary to vary this position higher or lower before the desired effect upon the curve can be attained. It is remarkable how plainly this action when correctly performed, affects the curve. In slight cases the spinous processes may be drawn completely in to a straight line at once and the projection of the ribs depressed towards a plane with the opposite side, showing that the vertebra are rotated into or towards their normal position. Of course, this effect only lasts as long as the muscles are held in contraction, but the value of the exercise is thus proved, and the practical result is eventually a permanent lessening or remoral of the rotation (Fig. 1 and Fig. 2).

In the exercise upon the front of the thorax (Fig. 3) the effect is less evident. The patient, in a sitting posture, draws the elastic cord forwards from a fixed point on or about a level with the shoulder on the side of the dorsal concavity, this point being situated behind the patient. This plan brings into use the muscles between the arm and the front of the thorax (the pectoral muscles), which thus tend to pull the front wall of the thorax, unduly prominent on this side, backwards. Here, as in the former instance, the surgeon must see that the effect is beneficial before he allows the exercise to be practised.

Figures 4 to 13 show some of the postures adopted in the various exercises in the recumbent position.

It would be almost impossible to give illustrations of all the exercises which may be used beneficially in the treatment of curvatures of the spine; they have to be varied to

meet the requirements of each individual case. Those given above will, however, afford a fair illustration of some of the more important positions produced in this treatment.

Queen Anne-street, W.

\section{CONSECUTIVE CASES OF ABDOMINAL SECTION IN HOSPITAL PRACTICE WITHOUT A DEATH.}

By WILLIAM DUNCAN, M.D. BRUX., F.R.C.S. Eng., OISSTETRIC PHYSICIAN TO THE MIDILLESEX MOSPITAL; SENIOR PHYSICIAN TO THE CHELSEA HOSPITAL FOR WOMEN.

SUCH a series of cases as that about to be given is sufficiently rare to merit publication; indeed, I am not aware that a similar series has occurred hitherto in this countryat all events it has not been recorded.

On Oct. 7th, 1897, a patient on whom I had operated for ruptured extra-uterine gestation at the Chelsea Hospital for Women died suddenly on the seventh day from pulmonary embolism when she seemed to be doing perfectly well. From that date up to now (June 10tb, 1900) all my sections at the above hospital are included in the following table. The cases, I may add, were the only ones admitted into my wards which required abdominal section. There was no choosing of favourable cases-indeed, the ninety-eighth case was about the worst of the series, being a suppurating ovarian cyst with pedicle tightly twisted three times, and the cyst wall was universally adherent, anteriorly to the parietal peritoneum and in the rest of its extent to the intestines, so intimately that the latter could only be freed by leaving a thin layer of the cyst wall adherent to them.

100 Abdominal Sections performed at the Chelsea Hospital for Women between Oct. $7 \mathrm{th}, 1897$, and June 10th, 1900.

Uterine fibroids - Suppurating broad ligamyomectomy

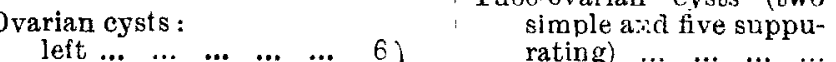
right double dermoids (one single and three double)

Ventro-fixation ...

Extra-uterine gestation: ruptured

$$
\text { unuptured }
$$

Py usalpinx (double) ... ...

Hydrosalpinx (one single and three double)

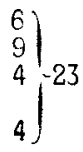
23 Diseased and adherent appendages : right appendage removed .. $\ldots \ldots \ldots$
left anpendage
releft anpent $\begin{array}{ccc}\operatorname{moved...} & \ldots \ldots & \ldots \\ \text { both appendages } & \text { re- }\end{array}$ mover adbesions $\cdots$ brolsen down $\ldots . . . \quad \ldots \quad \ldots$

Ventral bernia

Exploratory incisions Parovarian cysts...

In most of the cases of pyosalpinx and suppurating tuboovarian cysts the tumours were so kound down by adhesions that during removal they burst, allowing the purulent contents to escape into the peritoneal cavity. Whenever this occurred the abdomen was thoroughly flushed out with sterilised water. Ventro-fixation was performed either for old-standing prolapse or for retroflexion with adhesions binding the fundus down to the sacrum. The most difficult operations of the series were those for diseased and adherent appendages. The patients had had repeated attacks of peritonitis and they suffered more or less continual abdominal pain, so that they were chronic invalids. In these cases on opening the abdomen all the pelvic organs were usually found to be densely matted together ; the tubes and ovaries were matted to one another, to the pelvic wall, and to the intestines, so that usually the greatest difficulty was experienced in their removal. The one case of ventral hernia was in a woman on whom I had performed ovariotomy five years previously when the abdominal walls used to be sewn up with one layer of sutures. Nowadays, by sewing up the peritoneum, the rectal sheath, and the skin separately. ventral hernia.1s prevented. Exploratory incision without removal of any organ was performed three times. One case proved to be extensive malignant infiltration, the second was chronic cellulitis in the left broad ligament, and in a third a tumour of the size of an orange (probably malignant) was found to be growing in the posterior wall of the bladder. The results of abdominal section at the Chelsez Hospital 